\title{
Community-acquired pneumonia in children aged 2 months to 5 years: application of the WHO guidelines in a developed country setting (Switzerland)
} Received: 15 February 2002 / Revised: 16 April 2002 and 24 May 2002 / Accepted: 28 May 2002 / Published online: 6 July 2002
(C) Springer-Verlag 2002

In developed countries, the high morbidity associated with respiratory tract infections and the difficulty to discriminate viral from bacterial aetiology support the use of guidelines based on the observation of clinical signs, such as the World Health Organisation algorithm. Tachypnoea is the cardinal clinical sign on which early diagnosis of pneumonia is to be based in order to allow prompt initiation of appropriate antibiotic therapy.

A key issue in managing respiratory tract infections (RTI) is to detect cases of bacterial community-acquired pneumonia (CAP) requiring antibiotic treatment [1,2]. The only validated guideline in children is that proposed by the World Health Organisation (WHO) [6] which is based on the observation of five clinical parameters: respiratory rate (the absence of tachypnoea rules out CAP), chest retraction, cyanosis, feeding difficulty and absence of wheezing. Diverse combinations of these five parameters define three stages of severity, each calling for a distinct therapeutic regimen. In order to test the relevance of the WHO guidelines for a developed country, we applied them in our general paediatric emergency clinic in the light of routine investigations. In children with suspected CAP, after clearing the nose and when the child had returned to a quiet state, respiratory rate was counted for $1 \mathrm{~min}$ on two separate occasions. Tachypnoea was defined as any respiratory rate $>50 / \mathrm{min}$ in children younger than 1 year or $>40 / \mathrm{min}$ in children aged 1-5 years. All children aged 2 months to 5 years with WHO criteria for CAP were prospectively enrolled. Children on antibiotics or having received antibiotics within the previous 2 weeks, those with an underlying chronic disease and those with wheezing were excluded.

M. Dirlewanger $\cdot$ J-D. Krahenbuhl $\cdot$ S. Fanconi

B. Vaudaux $\cdot$ M. Gehri $(\square)$

Department of Paediatrics; Centre Hospitalier

Universitaire Vaudois, Hôpital de l'Enfance de Lausanne,

Bugnon 21, 1011 Lausanne, Switzerland

E-mail: Mario.Gehri@chuv.hospvd.ch

Tel.: +41-21-3143718

Fax: + 41-21-3143671
A chest X-ray film (antero-posterior and lateral views) was obtained in all children. White blood cell (WBC) count, C-reactive protein (CRP) and blood cultures were obtained in children with stage 2 and 3 pneumonia. Amoxyclavulanate was used for initial oral therapy (stage 1) and follow-up oral therapy (stages 2 and 3), and ceftriaxone for initial parenteral therapy (stages 2 and 3). Simple statistical analysis was done using the chi-squared test (level of significance defined as $P<0.05)$.

Over the 6 months study period, 47 children fulfilling the WHO criteria of pneumonia were enrolled. The median age was 28 months (range, 4 to 59 months). Seventeen children (36\%) were classified as stage 1 pneumonia, $22(47 \%)$ as stage 2 , and $8(17 \%)$ as stage 3 (Table 1). Clinical outcome was favourable in all children.

The overall poor sensitivity of auscultation, which proved normal in $70 \%$ of children is worth noting. Auscultation was able to detect only $33 \%$ of consolidations evidenced on chest films. Chest X-ray films proved to have an excellent sensitivity with abnormal findings in $98 \%$ of cases. A consolidation was evidenced in $92 \%$ of children meeting the WHO clinical criteria of pneumonia. In one child with stage 3 pneumonia and abnormal auscultatory findings, the chest X-ray film was normal on initial work-up but showed a consolidation $24 \mathrm{~h}$ later. This delay in the anatomical consolidation process has been well described in the literature [1]. We did not study the specificity of the chest X-ray examination (proportion of films showing a consolidation in children without tachypnoea). To our knowledge, only one investigation has specifically addressed the issue and came up with a value of $67 \%$ [5]. Pleural effusions were found in all three clinical stages of pneumonia. Overall, an effusion was detected in $17 \%$ of cases. It was more frequent in stage 3 pneumonia with $50 \%$ of children having some degree of pleural effusion. There were no statistically significant differences between groups, probably because numbers of subjects were insufficient to reach statistical significance. 
Table 1. Summary of results

\begin{tabular}{|c|c|c|c|c|c|}
\hline WHO stage & $\begin{array}{l}1 \\
17(36)\end{array}$ & $\begin{array}{l}2 \\
22(47)\end{array}$ & $\begin{array}{l}3 \\
8(17)\end{array}$ & Subtotal & $\begin{array}{l}\text { Total } \\
47\end{array}$ \\
\hline \multicolumn{6}{|l|}{ Auscultation } \\
\hline Normal $(n)(\%)$ & $13(76)$ & $16(73)$ & $4(50)$ & $33(70)$ & 47 \\
\hline Abnormal $(n)(\%)$ & $4(24)$ & $6(27)$ & $4(50)$ & $14(30)$ & \\
\hline \multicolumn{6}{|l|}{ Chest X-ray film } \\
\hline Normal $(n)$ & 0 & 0 & 1 & 1 & 47 \\
\hline Consolidation $(n)$ & 16 & 20 & 7 & 43 & \\
\hline Diffuse infiltrate $(n)$ & 1 & 2 & 0 & 3 & \\
\hline $\begin{array}{l}\text { Correlation between auscultation } \\
\text { and X-ray consolidation }(\%)\end{array}$ & 25 & 30 & 57 & 33 & \\
\hline Pleural effusion $(n)(\%)$ & $2(12)$ & $2(9)$ & $4(50)$ & $8(17)$ & \\
\hline WBC count $>15 \mathrm{~g} / \mathrm{l}(n)(\%)$ & NA & $9(41)$ & $7(88)$ & $16(53)$ & \\
\hline CRP level $>60 \mathrm{mg} / \mathrm{l}(n)(\%)$ & NA & $13(59)$ & $7(88)$ & $20(67)$ & \\
\hline $\begin{array}{l}\text { Blood culture positive for Streptococcus } \\
\text { pneumoniae }(n)(\%)\end{array}$ & NA & $1(5)$ & $3(38)$ & $2(12)$ & \\
\hline
\end{tabular}

Tachypnoea is as good a diagnostic criterion as the combination of clinical and radiological signs of consolidation. This finding does strongly question the use of routine chest $\mathrm{X}$-ray examination in the initial evaluation of children suspected to have a CAP. A WBC count above the cut-off point of $15 \mathrm{~g} / 1$ and CRP values greater than $60 \mathrm{mg} / 1$ [3] were found in $53 \%$ and $67 \%$ of patients with stage 2 or 3 pneumonia respectively. The proportion of cases with WBC counts and CRP values above the defined cut-off points was $88 \%$ in children with stage 3 pneumonia. In our opinion, the finding of elevated WBC counts and CRP levels does not provide much additional information and we consider these investigations to be of limited usefulness.

Investigating the microbial aetiology of RTIs was not the aim of this study. We limited our investigations to obtaining blood cultures in stage 2 and 3 pneumonia. They were positive for Streptococcus pneumoniae in 5\% of patients with stage 2 pneumonia as opposed to $38 \%$ of children with stage 3 disease. This suggests that the probability is low for blood culture results to have a practical impact on management. In our view blood cultures should not be obtained in stage 1 or 2 pneumonia. Further work should aim at validating the simple clinical approach of RTIs advocated by the WHO in view of specific causative pathogens in order to determine the proportion of viral infections being inappropriately treated with antibiotics, and at evaluating the diagnostic usefulness in this field of new biological markers of infection such as procalcitonin [4].

\section{References}

1. Coote N, McKenzie S (2000) Diagnosis and investigation of bacterial pneumonia. Paediatr Respir Rev 1: 8-13

2. Health PT (2000) Epidemiology and bacteriology of bacterial pneumonia. Paediatr Respir Rev 1: 4-7

3. McCracken Jr GH (2000) Etiology and treatment of pneumonia. Pediatr Infect Dis J 19: 273-277

4. Moulin F, Raymond J, Lorrot M, Gendrel D (2000) Procalcitonin in children admitted to hospital with community acquired pneumonia. Arch Dis Child 83: 320-324

5. Palafox $M$, Guiscafré $H$, Reyes $H$, Munoz $O$, Martinez $H$ (2000) Diagnostic value of tachypnoea in pneumonia defined radiologically. Arch Dis Child 82: 41-45

6. World Health Organisation (1994) Program for control of acute respiratory infections, 6th program report. WHO/ARI/94.33 